Research Paper

\title{
Time course of bone screw fixation following a local delivery of Zoledronate in a rat femoral model - A micro-finite element analysis
}

\author{
Ulrike Kettenberger ${ }^{1}$, Adeliya Latypova ${ }^{1}$, Alexandre Terrier, \\ Dominique P. Pioletti*
}

Laboratory of Biomechanical Orthopedics, Institute of Bioengineering, École Polytechnique Fédérale de Lausanne (EPFL), Switzerland

\section{A R T I C L E I N F O}

Article history:

Received 15 September 2014

Received in revised form

3 January 2015

Accepted 5 January 2015

Available online 19 January 2015

Keywords:

Drug delivery

Hydrogel

Bisphosphonate

Screw fixation

microCT

microFE

\begin{abstract}
A B S T R A C T
A good fixation of osteosynthesis implants is crucial for a successful bone healing but often difficult to achieve in osteoporotic patients. One possible solution to this issue is the local delivery of bisphosphonates in direct proximity to the implants, A critical aspect of this method, that has not yet been well investigated, is the time course of the implant fixation following the drug release. Usual destructive mechanical tests require large numbers of animals to produce meaningful results. Therefore, a micro-finite element (microFE) approach was chosen to analyze implant fixation. In vivo micro computed tomography (microCT) scans were obtained, first weekly and later bi-weekly, after implantation of polymeric screws in the femoral condyles of ovariectomized rats. In one half of the animals, Zoledronate was released from a hydrogel matrix directly in the peri-implant bone stock, the other animals were implanted only with screws as control. The time course of the implant fixation was investigated with linear elastic microFE models that were created based on in vivo microCT scans. The numerical models were validated against experimental pullout-tests measurements in an additional cadaver study. The microFE analysis revealed a significant increase in force at yield of the Zoledronate treated group compared to the control group. The force of the treated group was $28 \%$ higher after 17 days of screw implantation, $42 \%$ higher after 31 days. The significant difference persisted until the end of the in vivo study at day $58(p<0.01)$. The early onset and prolonged duration of the implant anchorage improvement that was found in this study indicates the great potential of Zoledronate-loaded hydrogel for an enhancement of osteosynthesis implant fixation in impaired bone.
\end{abstract}

c) 2015 Elsevier Ltd. All rights reserved.

\footnotetext{
*Correspondence to: EPFL/STI/IBI/LBO, Station 19, 1015 Lausanne, Switzerland. Tel.: +41 21693 8341; fax: +41 216938660.

E-mail address: dominique.pioletti@epfl.ch (D.P. Pioletti).

${ }^{1}$ Equally contributing.
} 


\section{Introduction}

The success of orthopedic implants is highly depending on their anchorage in surrounding bone. A good implant fixation requires a good structural integration between bone and implant surface (osteointegration) as well as a strong bone structure around the implant that can resist the loads transferred from the implant to the bone (Schiuma et al., 2013). Many successful strategies have been developed to improve the implant osteointegration such as surface topography changing treatments, osteoconductive coatings or surface functionalization with biological molecules (Zhang et al., 2014; Jäger et al., 2007). An improvement of the second aspect, the mechanical competence of the bone close to the implant, is however much more difficult to achieve. Due to the demographic changes in our population, more and more people suffer from age-related, bone impairing diseases such as osteoporosis (Hernlund et al., 2013). Affected patients have a significantly increased fracture risk, and once a fracture occurred, its treatment is challenging since osteosynthesis implants are difficult to anchor in the deteriorated bone structure (Broderick et al., 2013; Cummings and Melton 2002). High complication rates are therefore typical for osteoporotic fracture treatments and show the need for new strategies to enhance implant fixation in low quality bone. One of the most promising approaches to develop in recent years is the local delivery of anti-resorptive drugs such as bisphosphonates (BPs) in the periimplant bone. Many studies have shown that this strategy can locally enhance the bone density and therefore increase the mechanical stability of implants in animals and humans (Peter et al., 2006; Andersson et al., 2010; Gao et al., 2009; Abtahi et al., 2012). The improvement of implant anchorage was typically measured in most of the published studies with terminal ex vivo mechanical tests such as pullout, pushout test or torque testing. As each destructive mechanical test requires the sacrifice of a group of animals, typically not more than 1 or 2 time points were investigated (Peter et al., 2006; Andersson et al., 2010; Qi et al., 2012; Skoglund et al., 2004). As a consequence, only little is known about the time course of the BP effect on implant fixation. The temporal effect, however, is a very important aspect regarding the fact that osteosynthesis implants are placed to stabilize bone fractures. Good fracture reposition and stabilization are crucial for a successful fracture healing (Augat et al., 2005). Therefore the onset of the positive BP effect on screw fixation should ideally occur as soon as possible after implantation and persist until the fracture has completely healed. This aspect has so far not been evaluated.

One very promising approach to monitor implant fixation in vivo is a technique introduced by Wirth et al. that combines microCT imaging with a micro-finite element (microFE) analysis for the investigation of the stability of bone-implant constructs (Wirth et al., 2010). This group demonstrated an excellent correlation between microFE predicted pull-out strength and measured pull-out strength in a cadaveric ovine vertebra model. Stadelmann et al. applied this technique for the first time to timelapsed microCT scans from an in vivo rat model and obtained good correlations for the stiffness of the bone-screw construct (Stadelmann et al., 2013). The use of in vivo microCT data for the microFE offers the unique possibility to closely monitor implant stability with a significantly reduced number of animals.
In addition, important bone parameters affecting the implant fixation can be identified easily as the mechanical information gained with the microFE can be directly linked with bone parameters measured on the microCT scans (Wirth et al., 2011).

MicroFE analysis was used in this study to investigate the temporal effect of locally delivered Zoledronate, a potent BP, on the fixation of miniature screws in a rat femoral model of postmenopausal osteoporosis. The goal of this analysis was to determine if the local BP delivery can achieve the fast and durable enhancement of implant fixation that is needed for osteosynthesis implants in osteoporotic bone.

\section{Materials and methods}

The in vivo microcT data, that provided the basis for the microfE models, were obtained from an animal study published earlier by our group (Kettenberger et al., 2014). Since the miniature polymer screws used in the in vivo study were not suitable for biomechanical pullout tests, we performed a complementary cadaver study with aluminum screws on similar bone specimens and with similar screw geometry. This cadaver study provided the data to identify a suitable failure criterion for the microFE models.

\subsection{In vivo microCT study}

The animal experiments were approved by the local animal care and use committee (license no. 2508.1, EXPANIM, SCAV, Epalinges, Switzerland). Eight ovariectomized rats were implanted with radiopaque polyetheretherketone screws in both femoral condyles. In 4 animals that were randomly assigned to a first group (Zol-Gel group), the pre-drilled screw holes (diameter $1.2 \mathrm{~mm}$, depth $3.5 \mathrm{~mm}$ ) were filled with $5 \mu$ l of a commercially available hyaluronic acid hydrogel (Termira AuxiGelTM, Stockholm, Sweden) containing $1 \mu \mathrm{g} / \mathrm{ml}$ of Zoledronate (Art.-Nr. ALX430-153-0000, Enzo Life Sciences, Farmingdale, USA). The rats of the second group (Control group) received only screws. Both femurs of all animals were scanned with a special in vivo microCT for small rodents (Skyscan 1076, Bruker microCT, Kontich, Belgium) at day 3, 10, 17, 31, 45, and 58 after screw implantation. The used scanning parameters were published earlier (Kettenberger et al., 2014). Hydroxyapatite-polymer phantoms (diameter $4 \mathrm{~mm}$, Bruker microCT, Kontich, Belgium) with known bone mineral density served as references for the microCT calibration. Not all samples of this study could be used for the microFE study as some screw heads were broken during insertion. The final analyzed samples size in Zol-Gel group was $n=6$ for the first 4 measured time points, $n=3$ for the 5 th time point and $n=4$ for the last time point. One animal from the ZolGel group had to be euthanized after 4 scans due to a tumor and one scan from the 5th time point had to be removed due to motion artifacts. The sample size in the Control group was $n=6$ for all time points, therefore a total of 67 microCT scans were analyzed. All animals were sacrificed at the time of the last microCT scan. 


\subsection{Experimental pullout test}

Six adult female Sprague Dawley rat cadavers with a mean weight of $310 \pm 30 \mathrm{~g}$ were retrieved from another animal study (license no. 2567.a, EXPANIM, SCAV, Epalinges, Switzerland). The rats were lactating for 7 days at the time of sacrifice and therefore presented a significantly reduced bone density compared to normal animals (Miller and Bowman, 1998). The 12 femurs of the animals were dissected directly after sacrifice and kept frozen in saline until use. After thawing, holes with a length of $3.2 \mathrm{~mm}$ were drilled unicortically in the femoral condyles with a motorized dentist's drill (DEC 100, Nobelcare, Sweden). Custom-made aluminum screws (thread length $3 \mathrm{~mm}$, diameter $1.4 \mathrm{~mm}$ ) with a $20 \mathrm{~mm}$ long rod attached to them instead of a screw head were inserted manually in the pre-drilled holes (Fig. 1, left). Aluminum was chosen as screw material because this material has sufficient mechanical competence for the mechanical testing, while being artifact-free in the microCT. The inappropriate biocompatibility of aluminum however makes its in vivo use impossible.

The implanted specimens were wrapped in saline soaked gauze to keep them moist during the imaging and microCT scans. The scanning parameters, adapted to the aluminum screws, were the following: spatial resolution $18.4 \mu \mathrm{m}, 0.5 \mathrm{~mm}$ aluminum filter, voltage $60 \mathrm{kV}$, current $167 \mu \mathrm{A}$, exposure time $480 \mathrm{~ms}$, rotation step $0.4^{\circ}$, frame averaging 2 . The microCT was calibrated again with the reference phantoms to ensure a comparability of the results obtained from the in vivo and the cadaveric study.

After the scanning, the specimens were positioned in acrylic glass rings (inner diameter $8 \mathrm{~mm}$ ) with a guiding device that centered the screw in the ring before fixing them with dental cement (Technovit $\AA 3040$ powder, Heraeus Kulzer, Hanau, Germany) (Fig. 1, middle). This fixation technique was chosen in order to mimic the boundary conditions that were later used for the microFE study.

The pull-out test was performed with a tensile testing machine (ElectroPuls E3000, Instron, High Wycombe, England). The sample in the acrylic glass ring was placed under a plate with a $8 \mathrm{~mm}$-hole that was fixed to the base of the machine. The rod as prolongation of the screw was passed through the hole and fixed in a special gripper that was attached to the head of the tensile testing machine (Fig. 1, right). The head was moved up with a speed of $1 \mathrm{~mm} / \mathrm{min}$. Forces and displacements were recorded with a frequency of $50 \mathrm{~Hz}$ after a pre-load of $0.5 \mathrm{~N}$ was reached. Stiffness, force at yield, and force at pullout were determined for each sample from the resulting force-displacement curves, whereas the stiffness was defined as the slope of the linear part of the curve and the yield as the endpoint of the linear part where plastic deformation of the sample starts. All calculations were done with Matlab (Mathworks, Natick, USA).

\subsection{Image processing}

The image processing was identical for the in vivo study and the cadaver study, only the segmentation thresholds were adapted to the different screw materials and scanning parameters based on the phantom calibration to ensure a comparability of the results. The reconstruction of the projection images was done with NRecon and GPURecon Server (Bruker microCT, Kontich, Belgium) following a protocol that was published earlier (Kettenberger et al., 2014). Image processing after reconstruction was done with CTan (Bruker microCT, Kontich, Belgium) and Amira ${ }^{\circledR}$ (FEI Visualization Sciences Group, Burlington, USA).

The first step of the image processing was a reduction of the dataset size. A VOI, including the full diameter of the femur from $5 \mathrm{~mm}$ distal to $5 \mathrm{~mm}$ proximal to the screw, was manually defined on all scans using CTan. The background and the patella were then removed with an automated algorithm. The preprocessed datasets were loaded in Amira ${ }^{\circledR}$ for a further processing with a custom written script. A reference dataset with a height of $6 \mathrm{~mm}$ was created with the screw in the center and concentric regions measuring 3, 4, 5, 6, 7 and $8 \mathrm{~mm}$ in diameter around. All datasets were superpositioned on the reference dataset, ensuring an equal alignment and positioning of all screws for the microFE analysis (Fig. 2). In a next step, the datasets were transformed to the coordinate system of the reference dataset and resampled by a factor of 3 using the standard interpolation mode of Amira ${ }^{\circledR}$ to reduce computing costs. The new voxel size after resampling was $55.2 \mu \mathrm{m}$. A noise reduction median filter was applied after transformation and resampling to smooth the resulting images. In a next step, the images were segmented by assigning different gray values to screw, background, bone, and bone marrow in each region allowing a separate analysis of bone and microFE results in different distances from the screw (Fig. 2). The bone volume
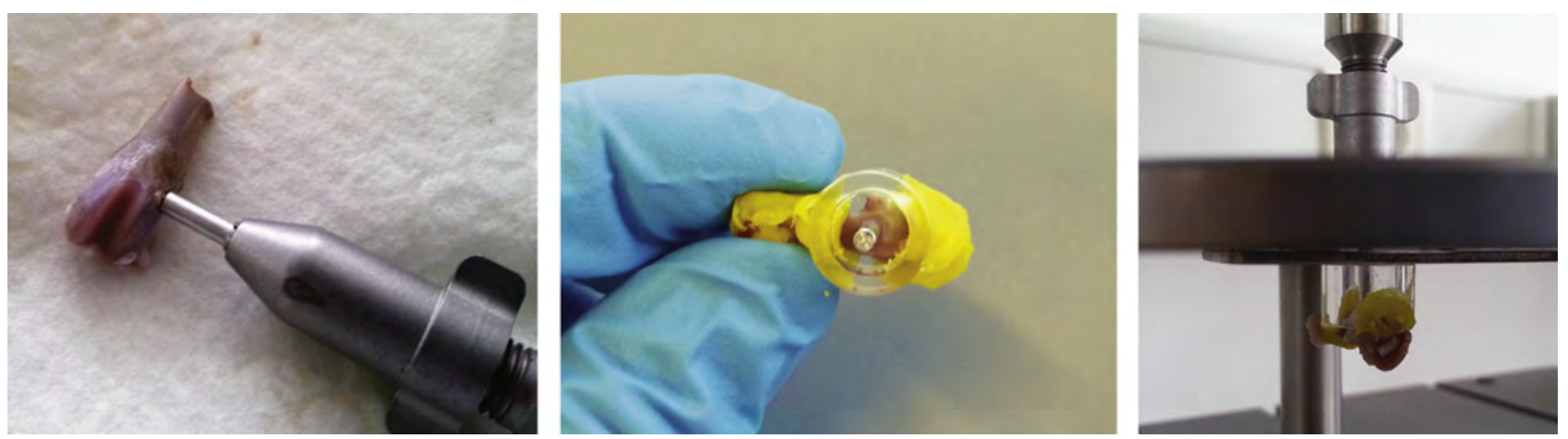

Fig. 1 - Preparation of the rat femurs for the pull-out test. Left: implantation of special aluminum screws in the femoral condyles. Middle: fixation of the specimen in acrylic glass rings with dental cement. Right: pull-out test performed with a tensile testing machine. 
A
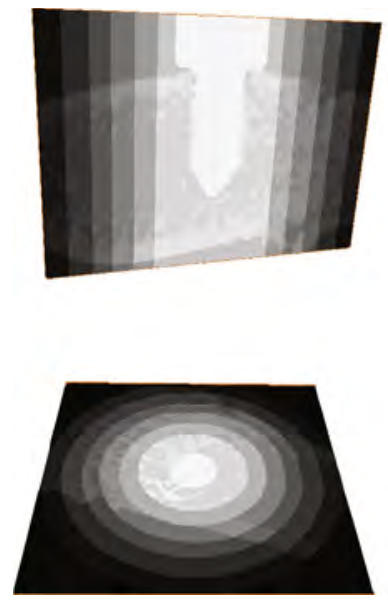

Processed MicroCT Scan
B
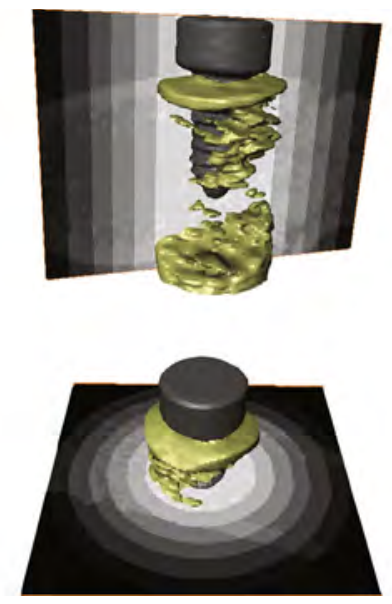

Analyzed Region: $\varnothing 3 \mathrm{~mm}$
C

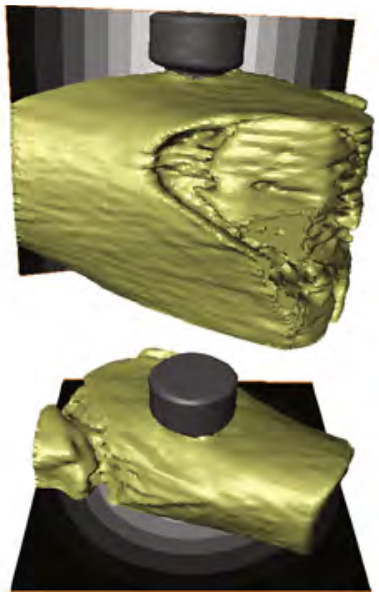

D

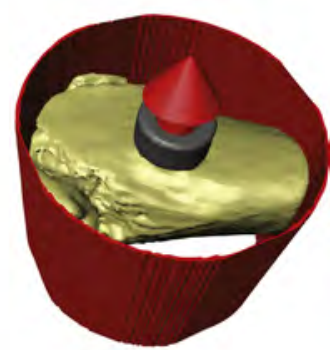

Simulation of the Pull-out:

-Cylindric Constraint of the Sample

- Vertical Screw Head Displacement

Fig. 2 - Image processing: processed microCT scan with centered screw and different gray values for concentric rings with diameters from 3 to $8 \mathrm{~mm}$ (A); bone within the $3 \mathrm{~mm}$ ring used for determining the bone volume fraction and the critical strain fraction (B) and full sample size with a diameter of $8 \mathrm{~mm}$ (C). MicroFE model of the pull-out test (D): the sample was constraint in a ring with $8 \mathrm{~mm}$ diameter, the screw head was displaced vertically along the screw axis.

fraction (BV/TV) including both cortical and cancellous bone was analyzed in the inner region with a diameter of $3 \mathrm{~mm}$ on the processed microCT scans.

\section{4. microFE analysis}

\subsubsection{Creation of the microFE models}

After image processing, linear elastic microFE models for all microCT scans of the in vivo and the cadaver study were created by converting image voxels to linear isotropic hexahedral finite elements (Muller and Ruegsegger, 1995). A cube of the same size as a microCT stack was created in the commercially available simulation software Abaqus. The cube was meshed with hexahedral elements whereas the edge length of one element in the model was equal to the edge length of one voxel in the pre-processed microCT datasets $(55.2 \mu \mathrm{m})$. An in-house Matlab script was used to assign mechanical properties to the elements according to the gray values in the microcT datasets. For the numerical models of the in vivo study we chose titanium as screw material as this is a common material for small bone screws and therefore gives more beneficial results than a model with PEEK screws. Different properties were given to bone $(E=6.8$ $\mathrm{GPa}, v=0.3$ ) (Stadelmann et al., 2013), bone marrow $(E=0.05$ $\mathrm{MPa}, v=0.3$ ) (Perren, 1979), the titanium screws of the in vivo study $(E=110 \mathrm{GPa}, v=0.32)$ and the aluminum screws of the cadaver study $(E=70 \mathrm{GPa}, v=0.35)$ (Stadelmann et al., 2013). No difference was made between the properties of cancellous and cortical bone as published before (Kabel et al., 1999). Elements surrounding the bone, which were identified as background during image processing, were removed from the model. The nodes of bone elements, located at the intersection with a virtual $8 \mathrm{~mm}$ ring around the screw, were fully constrained according to the sample fixation during the experimental pull-out test (Fig. 2). A displacement of $0.1 \mathrm{~mm}$ along the screw axis was applied to the top surface of the screw head in order to simulate the pullout test. The microFE simulations were performed on a cluster (Castor, EPFL, Switzerland) consisting of 50 nodes with 16 CPUs and 64 GB user memory each. We analyzed the reaction force on the screw head associated to the displacement, the resulting bone stiffness and the corresponding octahedral shear strain $\left(\gamma_{\text {oct }}\right)$ in the bone. Post-processing was done with an in-house python script.

\subsubsection{Identification of the failure criteria}

The failure criteria for the microFE models of the present study were identified based on the experimental results of the cadaver study. Therefore the experimentally measured forces at yield and pullout were compared with the microFE predicted reaction force at different strain states. Bone failure was assumed to occur when a certain bone volume fraction within a predefined VOI exceeded a critical strain level. Instead of using an effective strain criterion as has been done in other studies (Wirth et al., 2010; Stadelmann et al., 2013; Pistoia et al., 2002), we have chosen an octahedral shear strain criterion as it has been shown to be a good predictor for the spatial distribution of microdamage within trabecular bone (Nagaraja et al., 2005).

For the determination of the best failure criteria for forces at yield and pullout, we analyzed the microFE predicted reaction force at the points where a certain volume fraction of the bone within our pre-defined ROI (highly strained bone volume fraction) passed a certain level of $\gamma_{\text {oct }}$. To identify the best linear correlation, we analyzed combinations of 3 VOIs (diameter $3 \mathrm{~mm}, 4 \mathrm{~mm}$, and $5 \mathrm{~mm}$, height constant at $6 \mathrm{~mm}$ ), 3 levels of $\gamma_{\text {oct }}(0.5,0.7,1.0 \%)$ and 6 highly strained bone volume fractions (from 5 to 30\%). The analyzed strain range was chosen based on published values (Morgan et al., 2009; Carter et al., 1981). The combination that provided the highest coefficient of determination $\left(R^{2}\right)$ and the lowest $p$-value was chosen as critical strain level and critical highly strained bone volume fraction for a prediction of the force at yield and at pullout in the in vivo study. 
The experimentally measured stiffness from the cadaver study was compared to the microFE predicted stiffness to test for a linear correlation and to determine a scaling factor as microFE is known to significantly overestimate the boneimplant stiffness (Wirth et al., 2010).

\subsubsection{Analysis of the in vivo data}

After identification of the failure criteria and the correlations, the microFE models created from in vivo microCT scans were used to predict the force at yield and pullout as well as the bone-implant stiffness for all specimens at the 6 analyzed time points. Furthermore all possible correlations between BV/TV, stiffness, force at yield and force at pullout were examined for both the cadaver and the in vivo study. Finally, BV/TV from the cadaveric study was compared with the initial BV/TV of the in vivo Control group to test the comparability between the results.

\section{Statistical analysis}

The statistics analysis was done with Matlab. Significant differences between the Control group and the Zol-Gel group were assumed for a $p$-value smaller than 0.05 and tested with a Wilcoxon ranked sum test, as not all results were normally distributed.

\section{4. $\quad$ Results}

\subsection{Experimental pullout test}

Nine specimens were analyzed in total, 3 had to be excluded due to an atypical force-displacement curve without detectable yield that suggested that the screws were loose already at the beginning of the test. The average force at yield was $15.4 \pm 2.4 \mathrm{~N}$ (from $11.1 \mathrm{~N}$ to $20.1 \mathrm{~N}$ ), the average force at pullout was $21.6 \pm 4.0 \mathrm{~N}$ (from $13.0 \mathrm{~N}$ to $29.5 \mathrm{~N}$ ), and the average boneimplant stiffness was $107.6 \pm 25.7 \mathrm{~N} / \mathrm{mm}$ (from $59.2 \mathrm{~N} / \mathrm{mm}$ to $150.1 \mathrm{~N} / \mathrm{mm}$ ). A close inspection of the screws after pull-out did not show any damage to the screw threads.

\subsection{Identification of the failure criteria for the microFE} models

One microFE model was created for each experimentally analyzed cadaveric femur (9 models in total) and run successfully. The degrees of freedom in the models ranged from 2.1 to 3.1 million, requiring about $6 \mathrm{~h}$ of computing time for each sample. The coefficients of determination $\left(R^{2}\right)$ and $p$-values of all analyzed combinations of VOI, highly strained bone volume fraction and $\gamma_{\text {oct }}$ can be found in Table 1 for the force at yield and in Table 2 for the force at pullout.

For both analyzed forces, the best correlations between numerical predictions and experimental measurements were obtained for a highly strained bone volume fraction of $30 \%$ at an octahedral shear strain level of $0.5 \%$ measured within a cylindrical VOI with a diameter of $3 \mathrm{~mm}$. This finding was in agreement with a linear correlation between experimental force at yield and experimental force at pull-out $\left(R^{2}=0.887\right.$, $p=0.0002)$. The correlation between measured and predicted force at yield was better $\left(R^{2}=0.751, p=0.0025\right)$ than between measured and predicted force at pullout $\left(R^{2}=0.651, p=0.0086\right)$. Both predicted forces were overestimated by the numerical model by a factor of 6.1 and 6.8 (calculated based on the means of measured and predicted values). Therefore the predicted forces for the in vivo study were corrected based on the identified correlations (Fig. 3).

A significant, but weaker linear correlation was obtained for the measured and predicted bone-implant stiffness $\left(R^{2}=0.524, p=0.0275\right)$ (Fig. 4). The predicted stiffness was highly overestimated by a factor of 19.1, and again corrected based on the correlation for the in vivo study.

\subsection{Analysis of the in vivo study}

All 67 microFE models were created and analyzed successfully. Values for the force at yield and force at pullout, as well as for the bone-implant stiffness were predicted for all samples at all analyzed time points (Fig. 5).

The initial predicted force at yield was $16.4 \pm 1.1 \mathrm{~N}$ in the Control group and $16.1 \pm 1.0 \mathrm{~N}$ in the Zol-Gel group (Fig. 6 left).

Table 1 - Coefficients of determination $\left(R^{2}\right)$ and $p$-values for the linear correlation between the measured and predicted force at yield for different octahedral shear strain levels and different highly strained bone volume fractions measured in 3 VOIs. Empty cells mark combinations where the strain levels were not reached in the specified bone volume fraction (italic values: $p<0.01$ ).

\begin{tabular}{|c|c|c|c|c|c|c|c|c|c|c|c|c|c|}
\hline & \multirow{2}{*}{$\begin{array}{l}\gamma_{\text {oct }} \\
\text { level }\end{array}$} & \multicolumn{2}{|l|}{$5 \%$} & \multicolumn{2}{|l|}{$10 \%$} & \multicolumn{2}{|l|}{$15 \%$} & \multicolumn{2}{|l|}{$20 \%$} & \multicolumn{2}{|l|}{$25 \%$} & \multicolumn{2}{|l|}{$30 \%$} \\
\hline & & $\mathrm{R}^{2}$ & $p$ & $R^{2}$ & $p$ & $R^{2}$ & $p$ & $R^{2}$ & $p$ & $R^{2}$ & $p$ & $R^{2}$ & $p$ \\
\hline VOI & $0.5 \%$ & 0.576 & 0.0178 & 0.574 & 0.0181 & 0.594 & & 0.643 & 0.0151 & 0.726 & 0.0036 & 0.751 & 0.0025 \\
\hline$\varnothing$ & $0.7 \%$ & 0.567 & 0.0192 & 0.576 & 0.0178 & 0.597 & & 0.645 & 0.0147 & 0.733 & 0.0032 & - & - \\
\hline $3 \mathrm{~mm}$ & $1 \%$ & 0.571 & 0.0184 & 0.580 & 0.0172 & - & - & - & - & - & - & - & - \\
\hline VOI & $0.5 \%$ & 0.541 & 0.0238 & 0.540 & 0.0241 & 0.561 & & 0.590 & 0.0156 & 0.583 & 0.0166 & - & - \\
\hline$\varnothing$ & $0.7 \%$ & 0.539 & 0.0244 & 0.541 & 0.0238 & 0.561 & & - & - & - & - & - & - \\
\hline $4 \mathrm{~mm}$ & $1 \%$ & 0.541 & 0.0239 & 0.544 & 0.0233 & - & - & - & - & - & - & - & - \\
\hline VOI & $0.5 \%$ & 0.430 & 0.0222 & 0.563 & 0.0199 & 0.573 & & 0.548 & 0.0226 & - & - & - & - \\
\hline$\varnothing$ & $0.7 \%$ & 0.553 & 0.0217 & 0.563 & 0.0199 & 0.575 & & - & - & - & - & - & - \\
\hline $5 \mathrm{~mm}$ & $1 \%$ & 0.556 & 0.0211 & - & - & - & - & - & - & - & - & - & - \\
\hline
\end{tabular}


Table 2 - Coefficients of determination $\left(R^{2}\right)$ and $p$-values for the linear correlation between the measured and predicted force at pullout for different octahedral shear strain levels and different highly strained bone volume fractions measured in 3 VOIs. Empty cells mark combinations where the strain levels were not reached in the specified bone volume fractions (italic values: $p<0.01$ ).

\begin{tabular}{|c|c|c|c|c|c|c|c|c|c|c|c|c|c|}
\hline \multirow{3}{*}{$\begin{array}{l}\text { Force at } \\
\text { pullout }\end{array}$} & & \multicolumn{12}{|c|}{ Highly strained bone volume fraction } \\
\hline & \multirow{2}{*}{$\begin{array}{l}\gamma_{\text {oct }} \\
\text { level }\end{array}$} & \multicolumn{2}{|l|}{$5 \%$} & \multicolumn{2}{|l|}{$10 \%$} & \multicolumn{2}{|l|}{$15 \%$} & \multicolumn{2}{|l|}{$20 \%$} & \multicolumn{2}{|l|}{$25 \%$} & \multicolumn{2}{|l|}{$30 \%$} \\
\hline & & $\mathrm{R}^{2}$ & $p$ & $R^{2}$ & $p$ & $R^{2}$ & $p$ & $R^{2}$ & $p$ & $R^{2}$ & $p$ & $R^{2}$ & $p$ \\
\hline VOI & $0.5 \%$ & 0.423 & 0.0580 & 0.423 & 0.0580 & 0.448 & 0.0487 & 0.502 & 0.0326 & 0.592 & 0.0154 & 0.651 & 0.0086 \\
\hline$\varnothing$ & $0.7 \%$ & 0.413 & 0.0622 & 0.425 & 0.0572 & 0.450 & 0.0480 & 0.505 & 0.0320 & 0.605 & 0.0136 & - & - \\
\hline $3 \mathrm{~mm}$ & $1 \%$ & 0.417 & 0.0601 & 0.427 & 0.0561 & - & - & - & - & - & - & - & - \\
\hline VOI & $0.5 \%$ & 0.409 & 0.0636 & 0.416 & 0.0609 & 0.448 & 0.0485 & 0.500 & 0.0332 & 0.526 & 0.0270 & - & - \\
\hline$\varnothing$ & $0.7 \%$ & 0.405 & 0.0654 & 0.417 & 0.0603 & 0.449 & 0.0482 & - & - & - & - & - & - \\
\hline $4 \mathrm{~mm}$ & $1 \%$ & 0.406 & 0.0647 & 0.419 & 0.0594 & - & - & - & - & - & - & - & - \\
\hline VOI & $0.5 \%$ & 0.430 & 0.0553 & 0.459 & 0.0450 & 0.501 & 0.0337 & 0.507 & 0.0315 & - & - & - & - \\
\hline$\varnothing$ & $0.7 \%$ & 0.433 & 0.0540 & 0.458 & 0.0451 & 0.501 & 0.0328 & - & - & - & - & - & - \\
\hline $5 \mathrm{~mm}$ & $1 \%$ & 0.436 & 0.0528 & - & - & - & - & - & - & - & - & - & - \\
\hline
\end{tabular}
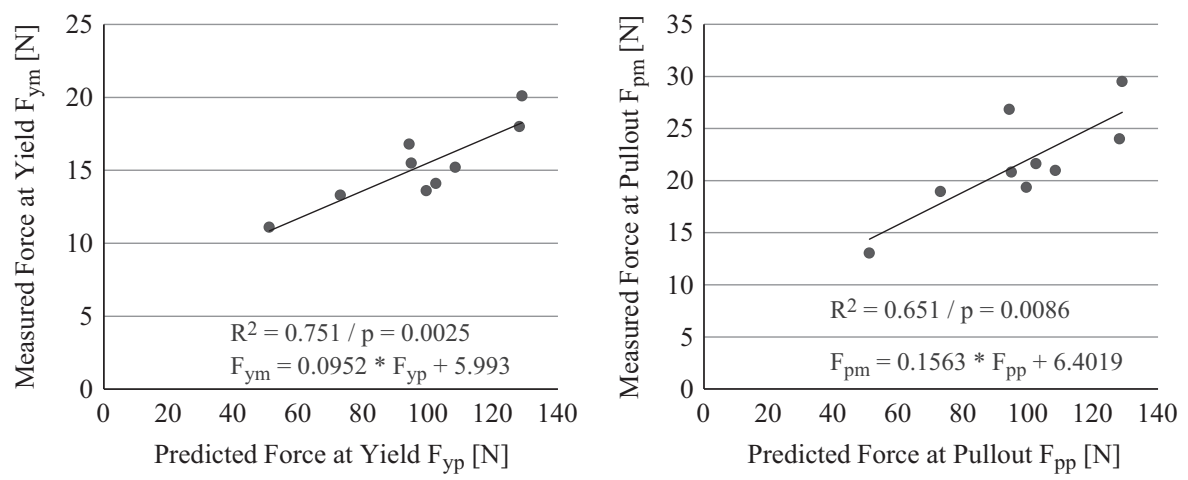

Fig. 3 - Correlation between measured and predicted force at yield (left), measured and predicted force at pullout (right); the experimental and numerical results were obtained from the rat cadaver study.

In the Control group it increased by $9 \%$ to $17.8 \pm 1.3 \mathrm{~N}$ between day 3 and day 10 and then did not show any marked changes until day 58. For the Zol-Gel group, however, a total increase in the predicted force at yield of $50 \%$ to $24.1 \pm 1.4 \mathrm{~N}$ was found between day 3 and day 31 followed by a slight decrease by $4 \%$ until day 58. The difference between the two groups was highly statistically significant starting from day 17 .

A similar time course was also found for the force at pullout (Fig. 6 right) and the predicted stiffness (Fig. 7) due to the correlations between all parameters. The force at pullout increased in the Control group from $23.4 \pm 1.7 \mathrm{~N}$ at day 3 by $10 \%$ to a maximum of $25.8 \pm 2.2 \mathrm{~N}$ at day 10 and did not change significantly any more until the end of the study. The Zol-Gel group started with a force at pullout of $23.0 \pm 1.6 \mathrm{~N}$ that increased in total by $57 \%$ up to $36.0 \pm 0.8$ at day 31 followed by slight decrease of $3 \%$ until day 58 . The initial value of the predicted stiffness was $145.5 \pm 12.7 \mathrm{~N} / \mathrm{mm}$ in the Control group and $147.3 \pm 17.8 \mathrm{~N} / \mathrm{mm}$ in the Zol-Gel group and rose by $16 \%$ to $169.9 \pm 23.0 \mathrm{~N} / \mathrm{mm}$ at day 58 in the Control group and by $55 \%$ to $229.1 \mathrm{~N} / \mathrm{mm}$ at day 45 in the Zol-Gel group. Again, a statistically significant difference was found between the groups starting from day 17.

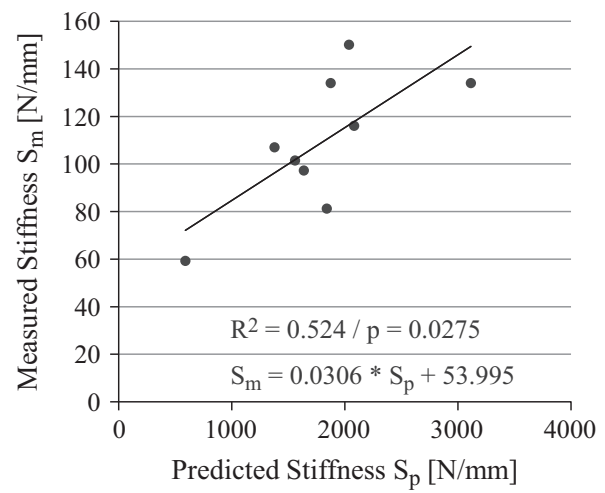

Fig. 4 - Correlation between measured and predicted boneimplant stiffness; the experimental and numerical results were obtained from the rat cadaver study.

The test for linear correlation of all measured parameters of the cadaver study revealed a strong linear correlation between force at yield and force at pullout $\left(R^{2}=0.886\right)$, weak correlations between BV/TV and force at yield $\left(R^{2}=0.468\right), B V / T V$ and stiffness 
Screw Head Displacement $0.03 \mathrm{~mm}$

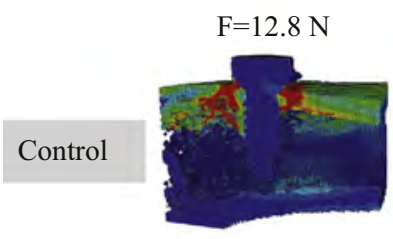

$\mathrm{F}=16.4 \mathrm{~N}$

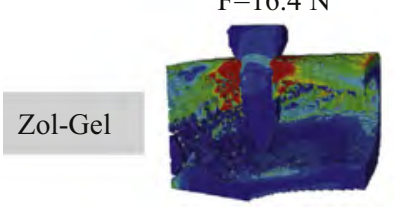

Day 3

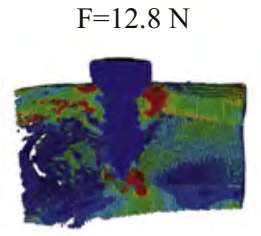

$\mathrm{F}=20.0 \mathrm{~N}$

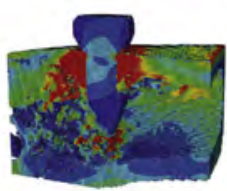

Day 10

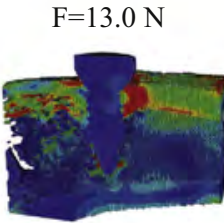

$\mathrm{F}=23.0 \mathrm{~N}$

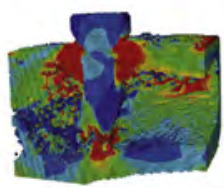

Day 17
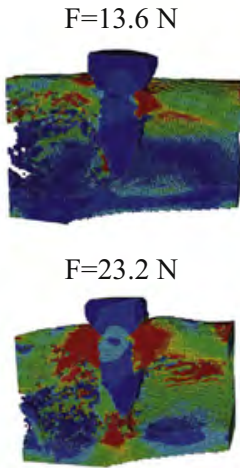

Day 31

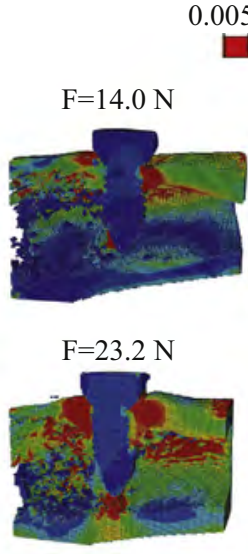

Day 45
$0.0025 \quad 0.000$

$\mathrm{F}=13.8 \mathrm{~N}$
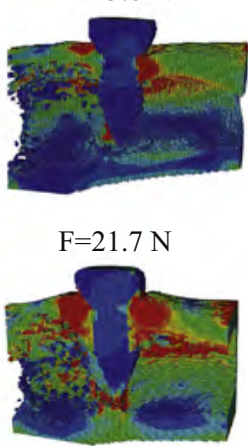

Day 58

Fig. 5 - One sample from each group showing the octahedral shear strain maps and corrected reaction forces at each time point determined for a screw head displacement of $0.03 \mathrm{~mm}$.
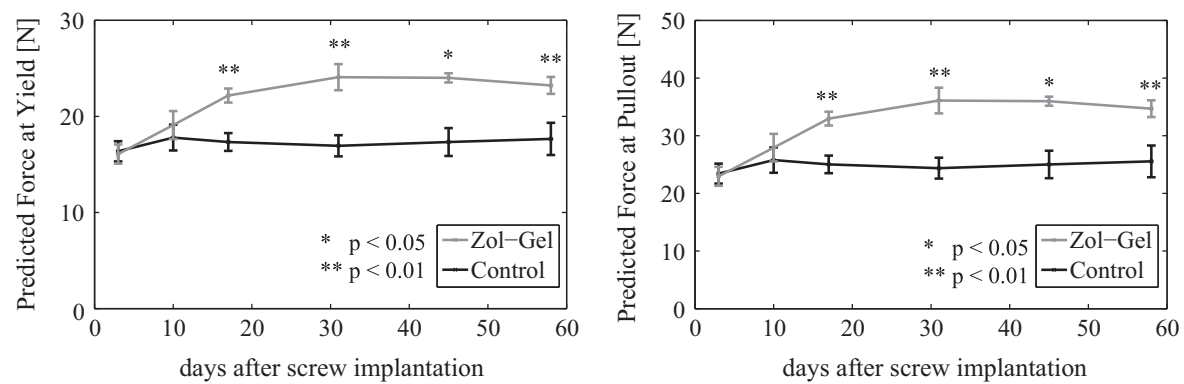

Fig. 6 - Time course of the predicted force at yield (left) and force at pullout (right) for the two groups of the in vivo study. The values were corrected based on the correlations identified in the cadaver study. The asterisks indicate significant differences between the two displayed groups.

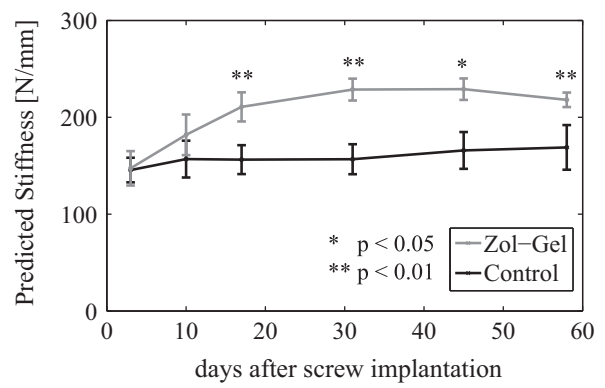

Fig. 7 - Time course of the predicted stiffness for the two experimental groups of the in vivo study. The values were corrected based on the correlations identified in the cadaver study. The asterisks indicate significant differences between the two displayed groups.

$\left(R^{2}=0.480\right)$, and force at yield and stiffness $\left(R^{2}=0.583\right)$. No correlation was found for the other combinations (Table 3). For the measured BV/TV and all predicted parameters of the in vivo study, we found high linear correlations between BV/TV and force at yield/pullout $\left(R^{2}=0.822\right)$ and stiffness and force at yield/ pullout $\left(R^{2}=0.862\right)$ and a lower correlation for BV/TV and stiffness $\left(R^{2}=0.671\right.$ ) (Table 3). As force at yield and force at pullout are determined based on the same reaction force from the model, their correlation is 1 and they have equal $R^{2}$ when compared with other parameters.

No significant difference was found between the BV/TV in the cadaver study and the initial BV/TV of the in vivo Controlgroup $(p=0.428)$.

\section{Discussion}

The goal of the present study was to investigate the temporal influence of locally delivered Zoledronate on the implant fixation in a rat model of postmenopausal osteoporosis. This was done based on time-lapsed in vivo microCT scans that were taken weekly to bi-weekly from screw implantation sites. The scans were used to create linear elastic microFE models, that could predict force at yield and pullout as well as stiffness of the bone-implant construct. With this microFE approach, we were able to confirm numerically the positive effect of locally delivered Zoledronate on implant fixation. More importantly, the combination of in vivo microCT with microFE analysis revealed insights in the time course of the Zoledronate effect showing, that a significant enhancement of screw fixation can be expected in rats as early as 17 days after implantation and can persist for minimum 6 weeks after onset. This result is of key importance considering the aim of 
Table 3 - Coefficients of determination $\left(R^{2}\right)$ and $p$-values for the linear correlations between all measured parameters of the cadaver study and the measured BV/TV and all predicted parameters of the in vivo study (underlined values: $p<0.05$, italic values: $p<0.01$.

\begin{tabular}{|c|c|c|c|c|c|}
\hline \multicolumn{2}{|c|}{ - Linear correlation with - } & \multicolumn{2}{|c|}{ Cadaver study $(n=9)$} & \multicolumn{2}{|c|}{ In vivo study $(n=67)$} \\
\hline & & $R^{2}$ & $p$ & $R^{2}$ & $p$ \\
\hline $\mathrm{BV} / \mathrm{TV}$ & Force at yield & 0.468 & 0.042 & 0.822 & 0.0000 \\
\hline $\mathrm{BV} / \mathrm{TV}$ & Force at pullout & $\overline{0.349}$ & $\overline{0.0942}$ & 0.822 & 0.0000 \\
\hline $\mathrm{BV} / \mathrm{TV}$ & Stiffness & 0.480 & 0.0386 & 0.671 & 0.0000 \\
\hline Stiffness & Force at yield & $\overline{0.583}$ & $\overline{0.0166}$ & 0.862 & 0.0000 \\
\hline Stiffness & Force at pullout & $\overline{0.378}$ & $\overline{0.0781}$ & 0.862 & 0.0000 \\
\hline Force at yield & Force at pullout & 0.886 & 0.0002 & 1 & 0.0000 \\
\hline
\end{tabular}

the local bisphosphonate delivery, which is the rapid stabilization of osteosynthesis implants in osteoporotic patients. The possibility for an early loading of the implant is crucial for an early mobilization of the patients. Therefore the drug effect should occur as soon as possible after implantation and persist until the fracture has healed completely.

A close investigation of the results from this study highlighted that the load in this femoral model of postmenopausal osteoporosis was mainly transmitted from the screw in the cortical bone as only little amounts of cancellous bone structure were left. The increase in force at yield and pullout as well as in stiffness for the Zol-Gel group was also caused to a high extent by the periosteal callus formation and resulting thickening of the cortex which was observed in this group around the screw head (Kettenberger et al., 2014). The newly formed trabecular bone seems to play a less significant role for the load transfer. No significant callus formation was observed in the control group which explains the small increase in predicted force at yield by only $9 \%$ occurring already during the first 10 days. Stadelmann et al. investigated the time course of the implant fixation in rat tibia without any pharmacological treatment (Stadelmann et al., 2013). They reported a time course similar to the one found in the present study for the force at yield with a peak at day 9, the reported maximum gain in force at yield however was much higher with $71 \%$ (Stadelmann et al., 2013). This significant difference might have been caused by the difference in screw size and geometry as well as by the intact trabecular bone structure of the healthy rats used in their study. The experimental data for force at yield and pullout, as well as for the stiffness, that were reported in this study, are generally low compared to published data from other rat studies (Andersson et al., 2010; Stadelmann et al., 2013). This can be explained by the experimental pullout test conditions of the present study including small screw size, the non-osteointegrated state of the screws and the low bone density of the rat cadavers used. When comparing the in vivo results of this study with previously published experimental data, excellent agreement can be found. Skoglund et al. performed pullout tests with miniature screws implanted in rat tibiae. They reported a 15\% larger force at pullout and $28 \%$ higher stiffness in animals that received miniature screws with a local dose of ibandronate compared to control 14 days after implantation (Skoglund et al., 2004). We found an increase in predicted force at pullout of $8 \%$ after 10 days and $32 \%$ after 17 days as well as an increase in stiffness of $16 \%$ after 10 days and $35 \%$ after 17 days for the
Zoledronate treated animals. Other studies obtained also similar values two weeks after screw implantation and bisphosphonate release (Tengvall et al., 2004; Wermelin et al., 2008). One more study from the Aspenberg group reported a $41 \%$ increase in pullout force for BP coated screws after 4 weeks (Agholme et al., 2011) what corresponds again very well with the $42 \%$ increase that we found after the same time period. We found only one long term study published by Wermelin et al. that investigated the temporal effect on screw fixation of pamidronate and ibandronate immobilized on stainless steel screws via a fibrinogen layer (Wermelin et al., 2007). For screws implanted close to the diaphyseal area in rat tibiae, a position comparable in terms of trabecular bone density to our OVX femoral model, they reported an increasing difference in pullout load between control and BP animals until week 4 (69\%) which diminished at week 8 (43\%). In the present study the peak difference between Control group and Zol-Gel group was also identified at week $4(42 \%)$ and slightly diminished at week 6 (39\%) and week 8 (31.5\%). Lower values in the present study might again be caused by smaller screws and the reduced trabecular bone density.

The screws used in the present in vivo study were made from a special barium sulfate-loaded PEEK that is visible on microCT scans but, unlike titanium or stainless steel implants, is relatively artifact-free. This way we eliminated one of the limitations of other microFE studies, which were based on in vivo microCT scans presenting metal artifact biased peri-implant bone structure (Wirth et al., 2010; Stadelmann et al., 2013). At the same time, the comparatively soft polymer screws did not allow us performing pullout tests for a validation of the model. Therefore we did a complementary study with rat cadavers for an identification of the possible correlations, which was designed to be as similar as possible to the actual in vivo study. The aluminum screws used for the cadaver study had an identical geometry as the PEEK screws of the in vivo study. Aluminum was chosen as screw material as it is relatively artifact-free in the microCT but nevertheless has sufficient mechanical properties for a pullout test. The bone loss situation was comparable in the lactating rats of the cadaver study and the OVX rats of the in vivo study as confirmed by comparison of the BV/TV. In the cadaver study we found a good linear correlation between predicted and measured force at yield $\left(R^{2}=0.75, p=0.003\right)$ and a weaker correlation for the force at pullout $\left(R^{2}=0.65, p=0.009\right)$ within a VOI of $3 \mathrm{~mm}$ diameter, with an octahedral shear strain level of $0.5 \%$ and a highly strained bone volume fraction of $30 \%$. A significant but weaker linear correlation was also found for 
the measured and predicted stiffness $\left(R^{2}=0.52, p=0.03\right)$. Wirth et al. and Stadelmann et al. also reported a good correlation for the failure load but did not find any correlation between predicted and measured stiffness in cadaver or ex vivo studies (Wirth et al., 2010; Stadelmann et al., 2013). One possible explanation for this difference is probably due to our osteoporotic model with a load transfer from the comparatively small screws mainly into the cortices.

The numerical models used for the present study were purely linear elastic, therefore only the linear elastic first part of the pullout test could be reliably predicted. The weak linear correlation that was found in this study between the measured and predicted force at pullout is caused by a linear correlation between the measured force at yield and the measured force at pullout. This linear correlation is not typical for pullout tests with bone screws (Stadelmann et al., 2013) and must be considered as being model-specific. Another limitation concerns the interface between screw and bone, which was considered as being fully bound in the numerical models. This assumption might be justifiable for fully osteointegrated screws but is questionable for screws directly after insertion or implanted into cadaveric bone. However, in screws unlike in pins, the threaded part of the screw achieves a form closure and prevents friction to have a decisive contribution to the pullout forces (Wirth et al., 2010; Stadelmann et al., 2013). The interface boundary condition, however, is most probably one of the main sources for the stiffness overestimation in our model. As already discussed by Wirth et al. (2010), some slipping between the screw and the bone, between the bone and the dental cement and some backlash in the experimental set-up is impossible to avoid. In our study, the small size of all components further complicated the experimental testing. Another factor that might have caused an overestimation of all predicted parameters, is the bone material property that was chosen based on the literature.

Additional bias of the predicted results is related to a down sampling of the element size from $18.4 \mu \mathrm{m}$ (microCT resolution) to $55.2 \mu \mathrm{m}$. This step was necessary to reduce computational costs, but it might have also distorted thin trabecular structures (Cooper et al., 2007). However, as mentioned above, the main loads in this osteoporotic model are taken by the cortices. Those are comparatively wide and therefore less sensitive to the bias that is caused by a reduction of the resolution. One further limitation that affects microcT based studies in general is the fact that computed tomography can detect the degree of mineralization of the bone but is unable to differentiate between mature lamellar bone and unstructured woven bone (Mulder et al., 2008). Woven bone can have a higher degree of mineralization than lamellar bone, but its irregular oriented collagen fibers and irregular mineralization patterns make it mechanically inferior (Currey 1998). The microFE approach considers all bone parts that exceed a certain mineralization degree (gray value threshold for bone segmentation) as equally mechanical competent. This might be a disadvantage in the current study as the bone trauma caused by the insertion of an implant is known to cause a rapid formation of woven bone in direct proximity of the implant (Marco et al., 2005). We recently showed that this early peri-implant bone formation is even enhanced by a fast local delivery of Zoledronate (Kettenberger et al., 2014). Studies have also shown that the BP can delay the remodeling of the newly formed woven bone to lamellar bone (McDonald et al., 2008), however the intrinsic mechanical properties of healing bone should not be affected by the BP (Amanat et al., 2008). All those processes and factors are not taken into account in the microFE analysis. For future studies, one possible improvement for this limitation could be the combination of dynamic histomorphometry (Kettenberger et al., 2014; Schulte et al., 2011) and microFE to identify newly formed bone. Depending on the maturity of the bone, the bone properties could then be adapted in the microFE model.

Despite several inherent limitations, the good correlations between the results of the present study and published experimental and numerical data confirm the great potential of microFE analysis to predict the time course of implant fixation in vivo and to assess how it is altered by the presence of the locally delivered Zoledronate.

\section{Conclusions}

With the present study, we were able to describe the time course of the Zoledronate effect on implant fixation in vivo based on microFE analysis. The locally delivered BP was predicted by the microFE model to significantly improve force at yield and pullout as well as stiffness already 2 weeks after screw implantation in a femoral rat model of postmenopausal osteoporosis. The difference between the groups persisted until the end of the study after 8 weeks. Those results indicate that the local delivery of bisphosphonate from a hydrogel matrix can improve the fixation of implants in impaired bone rapidly and over a long time period. These are very important aspects considering the fact that the bisphosphonate delivery system is intended to stabilize osteosythesis implant systems in osteoporotic bone.

\section{Acknowledgments}

Special thanks to Sandra Jaccoud at the LBO for her assistance during rat surgeries and dissection. This study was partially supported by a KTI grant (Project no. 11098.1).

\section{R E F E R E N C E S}

Abtahi, J., Tengvall, P., Aspenberg, P., 2012. A bisphosphonatecoating improves the fixation of metal implants in human bone. A randomized trial of dental implants. Bone 50, 1148-1151.

Agholme, F., Andersson, T., Tengvall, P., Aspenberg, P., 2011. Local bisphosphonate release versus hydroxyapatite coating for stainless steel screw fixation in rat tibiae. J. Mater. Sci. Mater. Med., 1-10.

Amanat, N., He, L.H., Swain, M.V., Little, D.G., 2008. The effect of zoledronic acid on the intrinsic material properties of healing bone: an indentation study. Med. Eng. Phys. 30, 843-847.

Andersson, T., Agholme, F., Aspenberg, P., Tengvall, P., 2010. Surface immobilized zoledronate improves screw fixation in rat bone: a new method for the coating of metal implants. J. Mater. Sci. Mater. Med. 21, 3029-3037. 
Augat, P., Simon, U., Liedert, A., Claes, L., 2005. Mechanics and mechano-biology of fracture healing in normal and osteoporotic bone. Osteoporos. Int. 16 (Suppl. 2), S36-S43.

Broderick, J.M., Bruce-Brand, R., Stanley, E., Mulhall, K.J., 2013. Osteoporotic hip fractures: the burden of fixation failure. Sci. World J. 2013.

Carter, D.R., Caler, W.E., Spengler, D.M., Frankel, V.H., 1981 Fatigue behavior of adult cortical bone: the influence of mean strain and strain range. Acta Orthop. Scand. 52, 481-490.

Cooper, D., Turinsky, A., Sensen, C., Hallgrimsson, B., 2007. Effect of voxel size on 3D micro-CT analysis of cortical bone porosity. Calcif. Tissue Int. 80, 211-219.

Cummings, S.R., Melton, L.J., 2002. Osteoporosis I: epidemiology and outcomes of osteoporotic fractures. Lancet 359, 1761-1767.

Currey, J.D., 1998. Mechanical properties of vertebrate hard tissues. Proc. Inst. Mech. Eng. Part H: J. Eng. Med. 212, 399-411.

Gao, Y., Luo, E., Hu, J., Xue, J., Zhu, S., Li, J., 2009. Effect of combined local treatment with zoledronic acid and basic fibroblast growth factor on implant fixation in ovariectomized rats. Bone 44, 225-232.

Hernlund, E., Svedbom, A., Ivergård, M., Compston, J., Cooper, C., Stenmark, J., et al., 2013. Osteoporosis in the European Union: medical management, epidemiology and economic burden: a report prepared in collaboration with the International Osteoporosis Foundation (IOF) and the European Federation of Pharmaceutical Industry Associations (EFPIA). Arch. Osteoporos., 8.

Jäger, M., Zilkens, C., Zanger, K., Krauspe, R., 2007. Significance of nano- and microtopography for cell-surface interactions in orthopaedic implants. J. Biomed. Biotechnol. 2007.

Kabel, J., Van Rietbergen, B., Dalstra, M., Odgaard, A., Huiskes, R., 1999. The role of an effective isotropic tissue modulus in the elastic properties of cancellous bone. J. Biomech. 32, 673-680.

Kettenberger, U., Ston, J., Thein, E., Procter, P., Pioletti, D.P., 2014. Does locally delivered Zoledronate influence peri-implant bone formation - spatio-temporal monitoring of bone remodeling in vivo. Biomaterials 35, 9995-10006.

Marco, F., Milena, F., Gianluca, G., Vittoria, O., 2005. Peri-implant osteogenesis in health and osteoporosis. Micron 36, 630-644.

McDonald, M.M., Dulai, S., Godfrey, C., Amanat, N., Sztynda, T., Little, D.G., 2008. Bolus or weekly zoledronic acid administration does not delay endochondral fracture repair but weekly dosing enhances delays in hard callus remodeling. Bone 43, 653-662.

Miller, S.C., Bowman, B.M., 1998. Comparison of bone loss during normal lactation with estrogen deficiency osteopenia and immobilization osteopenia in the rat. Anat. Rec. 251, 265-274.

Morgan, E.K., Yeh, T.M., Bone, O.C., 2009. Mechanics. In: Kutz, M. (Ed.), Biomedical Engineering and Design Handbook. McGrawHill, New York.

Mulder, L., Koolstra, J.H., Den Toonder, J.M.J., 2008. Van Eijden TMGJ. Relationship between tissue stiffness and degree of mineralization of developing trabecular bone. J. Biomed. Mater. Res. - Part A. 84, 508-515.

Muller, R., Ruegsegger, P., 1995. Three-dimensional finite element modelling of non-invasively assessed trabecular bone structures. Med. Eng. Phys. 17, 126-133.
Nagaraja, S., Couse, T.L., Guldberg, R.E., 2005. Trabecular bone microdamage and microstructural stresses under uniaxial compression. J. Biomech. 38, 707-716.

Perren, S.M., 1979. Physical and biological aspects of fracture healing with special reference to internal fixation. Clin. Orthop. Relat. Res. 138, 175-196.

Peter, B., Gauthier, O., Laib, S., Bujoli, B., Guicheux, J., Janvier, P., et al., 2006. Local delivery of bisphosphonate from coated orthopedic implants increases implants mechanical stability in osteoporotic rats. J. Biomed. Mater. Res. A 76, 133-143.

Pistoia, W., Van Rietbergen, B., Lochmüller, E.M., Lill, C.A., Eckstein, F., Rüegsegger, P., 2002. Estimation of distal radius failure load with micro-finite element analysis models based on three-dimensional peripheral quantitative computed tomography images. Bone 30, 842-848.

Qi, M., Hu, J., Li, J., Dong, W., Feng, X., Yu, J., 2012. Effect of zoledronate acid treatment on osseointegration and fixation of implants in autologous iliac bone grafts in ovariectomized rabbits. Bone 50, 119-127.

Schiuma, D., Plecko, M., Kloub, M., Rothstock, S., Windolf, M., Gueorguiev, B., 2013. Influence of peri-implant bone quality on implant stability. Med. Eng. Phys. 35, 82-87.

Schulte, F.A., Lambers, F.M., Kuhn, G., Muller, R., 2011. In vivo micro-computed tomography allows direct three-dimensional quantification of both bone formation and bone resorption parameters using time-lapsed imaging. Bone 48, 433-442.

Skoglund, B., Holmertz, J., Aspenberg, P., 2004. Systemic and local ibandronate enhance screw fixation. J. Orthop. Res. 22, 1108-1113.

Stadelmann, V.A., Conway, C.M., Boyd, S.K., 2013. In vivo monitoring of bone-implant bond strength by microCT and finite element modelling. Comput. Methods Biomech. Biomed. Eng. 16, 993-1001.

Tengvall, P., Skoglund, B., Askendal, A., Aspenberg, P., 2004. Surface immobilized bisphosphonate improves stainless-steel screw fixation in rats. Biomaterials 25, 2133-2138.

Wermelin, K., Tengvall, P., Aspenberg, P., 2007. Surface-bound bisphosphonates enhance screw fixation in rats - increasing effect up to 8 weeks after insertion. Acta Orthop. 78, 385-392.

Wermelin, K., Aspenberg, P., Linderback, P., Tengvall, P., 2008. Bisphosphonate coating on titanium screws increases mechanical fixation in rat tibia after two weeks. J. Biomed. Mater. Res. A 86, 220-227.

Wirth, A.J., Mueller, T.L., Vereecken, W., Flaig, C., Arbenz, P., Müller, R., et al., 2010. Mechanical competence of boneimplant systems can accurately be determined by imagebased micro-finite element analyses. Arch. Appl. Mech. 80, 513-525.

Wirth, A.J., Goldhahn, J., Flaig, C., Arbenz, P., Müller, R., Van Lenthe, G.H., 2011. Implant stability is affected by local bone microstructural quality. Bone 49, 473-478.

Zhang, B.G.X., Myers, D.E., Wallace, G.G., Brandt, M., Choong, P.F. M., 2014. Bioactive coatings for orthopaedic implants-recent trends in development of implant coatings. Int. J. Mol. Sci. 15, 11878-11921. 